P. Brekke, B. Fleck, and J. B. Gurman eds.

\title{
Evolution of the Filament and the Eruption of the Two-Ribbon Flare on MAY 13, 1981
}

\author{
A. A. $\mathrm{Xu}^{1}$, G. P. Wu ${ }^{2}$, Y. H. Tang ${ }^{1}$ \\ 1.Department of Astronomy, Nanjing University,Nanjing, China \\ 2.Department of Physics, Southeast University,Nanjing, China
}

\begin{abstract}
Using the observational $\mathrm{H} \alpha$, radio and $X$-ray data,we measure the ascending movement of the filament in the active region, and derive the current intensity and the current distribution inside the filament .The comparison between the ascending filament and the position of the hard X-ray image shows that they are co-spacial.These results suggest that the physical process in the thermal and non-thermal phases of the flare could be explained respectively by the tearing instability occurred inside the filament and the revised Alfvén-Carlqvist current interruption theory.The theoretical calculations are basically consistent with the observations.
\end{abstract}

\section{Several Important Observational Results}

The 3B/X1.5 class two-ribbon flare of 1981 May 13 took place in the Hale 17644 active region.It must be pointed out that an s-pattern $4.5 \times 10^{5} \mathrm{~km}$ long filament run through this active region and is well aligned with the magnetic neutral line .In order to explain theoretically the flare,we should address the following important results. (1)The flash phase of the flare occurred before the impulsive phase.There is a clear boundary between the thermal and nonthermal phases(Figure 1a)(Zhu et al.,1984;Tsuneta,1984); (2)Before and during the flare,the east-segment of the filament related to the flare were rising apparently ( Figure 1b); (3)The east segment of the ascending filament and the source of the X-ray flare are co-spacial and the latter runs in the direction of the former.

\section{The Theoretical Explanation of the Thermal Phase of the Flare on May 13,1981}

According to the above observational results,we consider that the occurrence of the flare is mainly due to the evolution of the filament current intensity in the active region.The rising of the filament implies the increase of the current intensity and the increase of the magnetic shear inside the filament.The latter one leads to the acceleration of the conversion of the magnetic energy into the rapid heating of the filament and its adjacent region through the resistive tearing instability. 

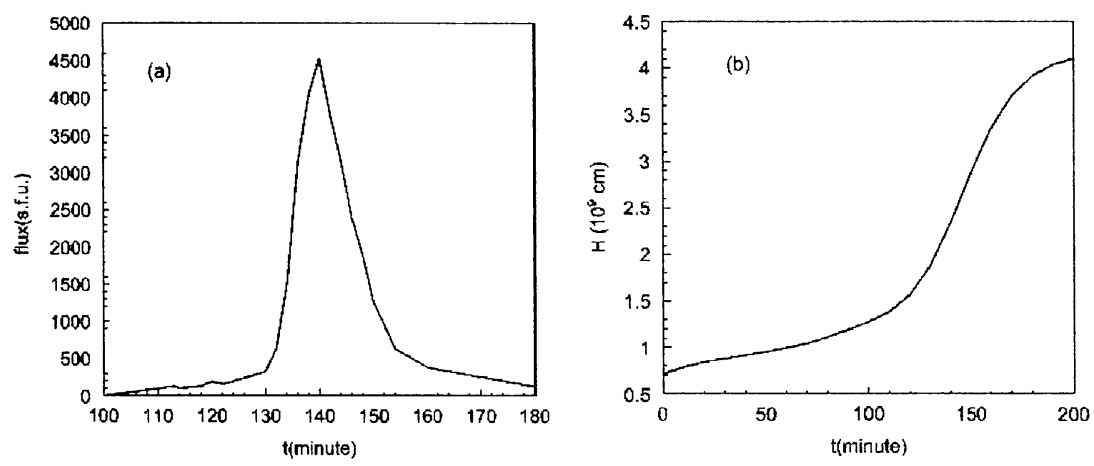

Figure 1. The time profiles of the microwave radioation of $3 \mathrm{~cm}$ (a) and the filament height (b). The abscissa represents the time in minute after 0200UT.

\section{1. the change of the current intensity and the magnetic shear inside the filament}

Using Kuperus-Raadu filament model and the $h-t$ curve(Figure 1b), one can calculate the evolution of the filament current intensity(Figure 2a). It's shown that the current intensity of the ascending filament increases with time and the profile of the $d I / d t$ (Figure 2(b)) is similar to the one of the radio emission(Figure $1(\mathrm{a})$ ).

Adopting the Lundquist field to express the force-free field inside the filament,we obtain the relationship between the current intensity and the magnetic shear inside the filament.The calculated force-free factor $\alpha(t)$ (Figure 2(c)) curve indicates that the magnetic shear inside the ascending filament increases with time.

\section{2. the magnetic energy could be converted into thermal energy through the resistive tearing instability}

When the magnetic shear inside the filament increases,the multiple tearing modes may take place(Spicer,1981). They provides the basic condition for conversion of the magnetic energy into heating the flare plasma through the resistive teraring instability. We can calculated the $T_{c}-t$ curve which shows the change of the temperature with time inside and near the filament (Figure $2(\mathrm{~d}))(\mathrm{Xu}$ et al.,1994).This result coincides with the observational result of the microwave radiation in the thermal phase and the soft X-ray radiation.

\section{The Physical Mechanism of the Non-thermal Phase of May 13 Flare}

The main observational results of nonthermal phase are as follows(Tsuneta,1984): (1) Both the microwave source and X-ray source were located stable at the top of $H_{\alpha}$ flare loop (about $4-5 \times 10^{4} \mathrm{~km}$ ); (2)There was a very broad population of non-thermal electrons. The microwave radiation and X-ray emmision of the 

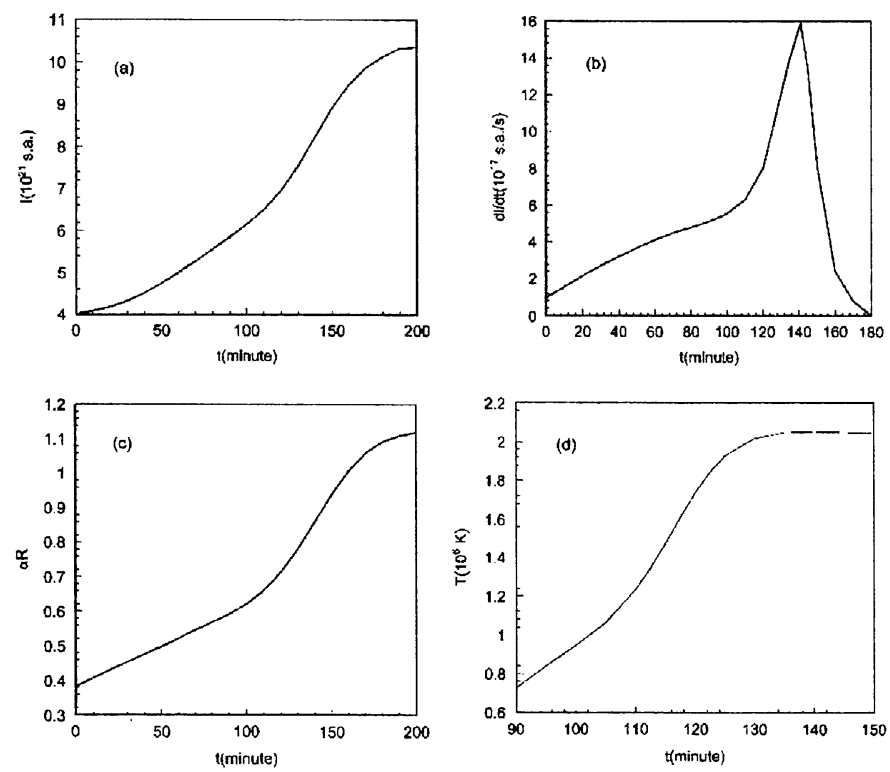

Figure 2. The theoretical results of (a) the time profile of the current intensity of the filament,(b)the differential curve of the current intensity (c)the time profile of the force-free factor $\alpha$, (d)the time profile of the temperature in the region of the flare source.The abscissa represents the time in minute after 0200UT.

flare mainly came from the non-thermal electrons; (3)Considering the fact that the position of the X-ray source is stable and the II-type radio burst was not found,we conclude that the non-thermal electrons could not be accelerated by II-type shock wave; (4) The growth rate of the intensity of the filament current suddenly increased by 3 times at 0405UT(seen in Figure 2(a)).Generally speaking,the fast increase of the current may lead to a strong current density near the filament surface.

The basic characteristics of the non-thermal phase suggest that the electron acceleration may be decribed by revised Alfvén-Carlqvist model (Spicer,1981). Using this model,the number of electrons accelerated by Drecier field is about $10^{35}-10^{36}$ (Xu et al,1994).

Because the hard X-ray source is located on the top of the loop,we may work out the numbers of electrons responsible for the observed hard X-ray bremsstrahlung in thin-target models(Hudson et al.1978). Using the flux of hard X-ray in the three different moments,we obtain the number of non-thermal electrons(seen in table 1). This shows that the theoretical result is consistent with the observation and proves that our explaination is reasonable.

This study was supported by Major Project 19791090 of National Science Foundation of China. 
Table 1. The number of the non-thermal electrons calculated from the flux of the x-ray

\begin{tabular}{|c|c|c|c|}
\hline time & $\gamma$ & $\Phi_{20}$ & $N_{20} \times 10^{34}$ \\
\hline $4: 12.45-50$ & 3.86 & 13.53 & 4.79 \\
\hline $4: 15.40-42$ & 3.57 & 42.22 & 14.15 \\
\hline $4: 26.00-10$ & 3.02 & 2.025 & 0.6345 \\
\hline
\end{tabular}

\section{References}

Hudson,H.S.,Canfield,R.C. and Kane,S.R.:1978,Solar Phys., 60, 137.

Spicer,D.S.:1981,Solar Phys., 70, 149.

Tsuneta,S.,Takakura,T. and Nitta,N.:1984,Ap.J.,280, 887.

$\mathrm{Xu}$ Aoao ,Wu Guiping and Tang Yuhua et al.:1994,Chinese Science Bulletin, 39, 759.

Zhu Zuyan,Wu Hongao and Cao Tianjun:1984,Acta Astronomica Sinica, 25,401. 